\title{
SOME NEW PROPERTIES OF TRANSFINITE ORDINALS ${ }^{1}$
}

\section{SEYMOUR SHERMAN}

1. Introduction. Sierpiński ${ }^{2}$ has stated the following theorem on the Cantor normal form:

Every ordinal $A>0$ can be represented in the form

$$
A=\sum_{i=0}^{n-1} \omega^{\alpha i} a_{i},
$$

where

$$
\begin{aligned}
& \omega>n, a_{0}, a_{1}, a_{2}, \cdots, a_{n-1}>0, \\
& A \geqq \alpha_{0}>\alpha_{1}>\alpha_{2}>\cdots>\alpha_{n-1} \geqq 0 .
\end{aligned}
$$

Henceforth in this paper, when an ordinal is written in summation form, it will be assumed that the summation satisfies the above-stated requirements.

Elsewhere Sierpiński ${ }^{3}$ has proved:

The set of all "divisors on the left" of an ordinal number is closed."

One of the purposes of this paper is to analyze further, by means of the Cantor normal form, the left and right factors of transfinite ordinals. In addition the Cantor normal form will be used to prove that for ordinals $A, B$, and $C$

$$
(A+B) C \leqq A C+B C .
$$

We can also specify the necessary and sufficient condition that the equality hold. F. Siecza ${ }^{5}$ has used the Cantor normal form to discuss

1 Presented to the Society, December 27, 1939. The author is indebted to Professor W. A. Hurwitz for his indispensable advice in the preparation of this paper.

${ }^{2}$ W. Sierpiński, Leçons sur les Nombres Transfinis, Paris, 1928, p. 202; hereafter referred to as Sierpinski (I). The theorem was first stated for ordinals of the first and second ordinal class by G. Cantor, Beiträge zur Begründung der transfiniten Mengenlehre, Mathematische Annalen, vol. 49 (1897), p. 237, and for all ordinals by G. Hessenberg, Grundbegriffe der Mengenlehre, Abhandlungen der Fries'schen Schule, New Series 1.4, Göttingen, 1906, p. 587.

${ }^{3} \mathrm{~W}$. Sierpinski, A property of ordinal numbers, Bulletin of the Calcutta Mathematical Society, vol. 20 (1930), pp. 21-22; hereafter referred to as Sierpinski (II).

${ }^{4} \mathrm{~A}$ set of ordinal numbers is said to be closed if it contains the least upper bound of each subset.

${ }^{5} \mathrm{~F}$. Siecza, Sur l'unicité de la décomposition de nombres ordinaux en facteurs irreductibles, Fundamenta Mathematicae, vol. 5 (1924), pp. 172-175. 
factorization of transfinite ordinals, but his problem was different from the one considered here.

2. Left and right factors. We shall now consider left factors.

2.1. Theorem. A necessary and sufficient condition that $x$ be a left factor of $A=\sum_{i=0}^{n-1} \omega^{\alpha_{i}} a_{i}$ is that either $0<x<\omega^{\alpha_{n-1}}$ or $x=\omega^{\alpha_{j}} p_{j}$ $+\sum_{i=j+1}^{n-1} \omega^{\alpha_{i}} a_{i}$, where $p_{j}$ is a factor of $a_{j}$ and $0 \leqq j \leqq n-1$.

If $0<x<\omega^{\alpha} n-1$, let $x=\sum_{i=0}^{m-1} \omega^{\beta_{i}} b_{i}$. Thus $\beta_{0}<\alpha_{n-1}$ and $-\beta_{0}+\alpha_{j}$, $0 \leqq j \leqq n-1$, exists. ${ }^{6}$ We have

$$
\left\{\sum_{i=0}^{m-1} \omega^{\beta_{i}} b_{i}\right\}\left\{\sum_{i=0}^{n-1} \omega^{-\beta_{0}+\alpha_{i}}\right\}=\sum_{i=0}^{n-1} \omega^{\alpha_{i}} a_{i},
$$

and hence $x$ is a left factor of $A$.

If $x=\omega^{\alpha_{i}} p_{j}+\sum_{i=j+1}^{n-1} \omega^{\alpha} a_{i}$, where $a_{j}=p_{j} \lambda_{j}$, then

$$
\begin{aligned}
\left\{\omega^{\alpha_{j}} p_{j}+\sum_{i=j+1}^{n-1} \omega^{\alpha_{i}} a_{i}\right\}\left\{\sum_{i=0}^{j-1}\right. & \left.\left(\omega^{-\alpha_{j}+\alpha_{i}}\right) a_{i}+\lambda_{j}\right\} \\
& =\sum_{i=0}^{j-1}\left(\omega^{\alpha_{j}-\alpha_{j}+\alpha_{i}}\right) a_{i}+\omega^{\alpha_{j}} p_{j} \lambda_{j}+\sum_{i=j+1}^{n-1} \omega^{\alpha_{i}} a_{i} \\
& =\sum_{i=0}^{j-1} \omega^{\alpha_{i}} a_{i}+\omega^{\alpha_{i}} a_{j}+\sum_{i=j+1}^{n-1} \omega^{\alpha_{i}} a_{i}=\sum_{i=0}^{n-1} \omega^{\alpha_{i}} a_{i}
\end{aligned}
$$

and $x$ is a left factor of $A$.

Thus we have proved the sufficiency of the condition.

Suppose that $x=\sum_{i=0}^{m-1} \omega^{\beta_{i}} b_{i}$ is a left factor of $A$ and that $x \geqq \omega^{\alpha-1}$. Then

$$
A=\left(\sum_{i=0}^{m-1} \omega^{\beta i} b_{i}\right)\left(\sum_{i=0}^{l-1} \omega^{\gamma_{i} c_{i}}\right)
$$

If $\gamma_{l-1}=0$ then

$$
\begin{aligned}
\sum_{i=0}^{n-1} \omega^{\alpha_{i}} a_{i} & =\left(\sum_{i=0}^{m-1} \omega^{\beta_{i}} b_{i}\right)\left(\sum_{i=0}^{l-1} \omega^{\gamma i} c_{i}\right) \\
& =\sum_{i=0}^{l-2}\left(\omega^{\beta_{0}+\gamma_{i}}\right) c_{i}+\omega^{\beta_{0}} b_{0} c_{l-1}+\sum_{i=1}^{m-1} \omega^{\beta_{i}} b_{i}
\end{aligned}
$$

where the last expression is a Cantor normal form. Comparing coefficients in the right and left ends of the series of equations, we see that

${ }^{6}$ See F. Hausdorff, Mengenlehre, 1935, p. 63. 
for some $j, 0 \leqq j \leqq n-1, \beta_{0}=\alpha_{j}, b_{0}$ is a factor of $a_{j}$, and $\sum_{i=1}^{m-1} \omega^{\beta_{i}} b_{i}$ $=\sum_{i=j+1}^{n-1} \omega^{\alpha_{i}} a_{i}$.

If $\gamma_{l-1}>0$, then

$$
\sum_{i=0}^{n-1} \omega^{\alpha_{i}} a_{i}=\left(\sum_{i=0}^{m-1} \omega^{\beta_{i}} b_{i}\right)\left(\sum_{i=0}^{l-1} \omega^{\gamma^{i}} c_{i}\right)=\sum_{i=0}^{l-1}\left(\omega^{\beta_{0}+\gamma_{i}}\right) c_{i} .
$$

But since $x \geqq \omega^{\alpha}{ }^{n-1}$, we have $\beta_{0} \geqq \alpha_{n-1}, \beta_{0}+\gamma_{l-1}>\alpha_{n-1}$, and thus the left and right ends of series of equations could not be equal. From this contradiction $\gamma_{l-1}=0$ and by the preceding argument our condition is necessary.

Since in $\sum_{i=0}^{n-1} \omega^{\alpha_{i}} a_{i}$ there is only a finite set of coefficients and since each of these coefficients, being finite, has only a finite set of factors, there is but a finite set of left factors of the form $\omega^{\alpha_{i}} p_{j}+\sum_{i=j+1}^{n-1} \omega^{\alpha_{i}} a_{i}$, $p_{j}$ a factor of $a_{j}$; that is, there is only a finite set of left factors greater than or equal to $\omega^{\alpha-1}$. Hence we have proved:

2.2. Corollary. The set of left factors of $A=\sum_{i=0}^{n-1} \omega^{\alpha} a_{i}$ consists of the set of all positive ordinals less than $\omega^{\alpha-1}$ and a finite set of ordinals not less than $\omega^{\alpha}{ }_{n-1}$.

2.3. Corollary. If $A=\sum_{i=0}^{n-1} \omega^{\alpha} a_{i}$, the left factors of $A$, in order of magnitude, form a normally ordered set whose ordinal number is $-1+\omega^{\alpha}{ }^{n-1}+\sum_{i=0}^{n-1} \tau\left(a_{i}\right)$, where $\tau\left(a_{i}\right)$ is the number of factors of $a_{i}$.

2.31. CoRollary. The ordinal number of the normally ordered set of left factors of a positive ordinal is either a finite ordinal or the sum of an additively indecomposable ordinal and a finite ordinal.

We can now obtain the previously stated result of Sierpinski, ${ }^{8}$ who makes no use of the Cantor normal form:

2.4. Corollary. The left factors of $A$ form a closed set.

The following corollary has been proved by Hausdorff ${ }^{9}$ and Bennet $^{10}$ by means of the euclidean algorithm.

2.5. Corollary. Any two ordinals greater than 0 have a greatest common left factor.

We shall prove this by means of the preceding corollary. Let $A>0$

${ }^{7}$ An ordinal $\alpha>0$ is said to be additively indecomposable when there do not exist ordinals $\beta, \gamma$ such that $\beta<\alpha, \gamma<\alpha$, and $\alpha=\beta+\gamma$. See Sierpinski (I), p. 178.

${ }^{8}$ Sierpiński (II).

๑ F. Hausdorff, Grundzïge der Mengenlehre, Leipzig, 1914.

10 A. A. Bennett, Some arithmetic operations with transfinite ordinals, American Mathematical Monthly, vol. 28 (1921), pp. 427-430. 
have a set of left factors, $\mathfrak{A}$, and $B>0$ have a set of left factors $\mathfrak{B}$. $\mathfrak{A} \mathfrak{B}$, the intersection of $\mathfrak{A}$ and $\mathfrak{B}$, has at least one element, 1 . The least upper bound, $\mathfrak{c}$, of $\mathfrak{A} \mathfrak{B}$ is a member of $\mathfrak{A}$ and a member of $\mathfrak{B}$, since both $\mathfrak{A}$ and $\mathfrak{B}$ are closed. Hence $\mathfrak{c}$ is a member of $\mathfrak{A} \mathfrak{B}$; that is, $\mathfrak{Y} \mathfrak{B}$ has a maximum element. Thus $\mathrm{c}$ is the greatest common left factor of $A$ and $B$.

The development in case of right factors is somewhat analogous. The following can easily be seen to be true.

2.6. THEOREM. If $A=\sum_{i=0}^{n-1} \omega^{\alpha_{i}} a_{i}$, then a necessary and sufficient condition that $x$ be a right factor of $A$ is that either $x=\sum_{i=0}^{n-1}\left(\omega^{-\beta_{0}+\alpha_{i}}\right) a_{i}$, where $\beta_{0}<\alpha_{n-1}$, or $x=\sum_{i=0}^{j-1}\left(\omega^{-\alpha_{j}+\alpha_{i}}\right) a_{i}+r_{j}$, where $r_{j}$ is a factor of $a_{j}$.

Since each exponent of the Cantor normal form has a finite set of residues and each coefficient has only a finite set of factors, we can state the following: ${ }^{11}$

2.7. TheOREM. The set of all right factors of any positive ordinal is finite.

Sierpiński has proved some of these theorems by using merely the properties of additively indecomposable ordinals. The use of the Cantor normal form not only yields more extensive results in the case of left factors, but also provides a unified approach to other problems in the theory of transfinite ordinals.

3. The distributive laws. Among transfinite ordinals the distributive law is always valid for one order of multiplication

$$
C(A+B)=C A+C B,
$$

but not always for the other order of multiplication

$$
(A+B) C \quad \text { ? } A C+B C \text {. }
$$

We shall prove:

3.1. Theorem. $(A+B) C \leqq A C+B C$.

If either $A, B$, or $C$ is zero, then our theorem holds. Consider $A>0$, $B>0$, and $C>0$, where

$$
\begin{gathered}
A=\sum_{i=0}^{n-1} \omega^{\alpha_{i}} a_{i}=\omega^{\alpha 0} a_{0}+A^{\prime}, \quad B=\sum_{i=0}^{m-1} \omega^{\beta_{i}} b_{i}=\omega^{\beta_{0}} b_{0}+B^{\prime}, \\
C=\sum_{i=0}^{l-1} \omega^{\gamma_{i}} c_{i} .
\end{gathered}
$$

${ }^{11}$ Sierpiński (I). 
$C$ is either finite, transfinite and limiting, or transfinite and nonlimiting.

Suppose $C$ is finite and $0<C<\omega$. If $\alpha_{0}<\beta_{0}$, then

$$
A+B=A+\omega^{\beta_{0}} b_{0}+B^{\prime}=B, \quad(A+B) C=B C=A C+B C .
$$

If $\alpha_{0}=\beta_{0}$, then

$$
A+B=\omega^{\beta_{0}}\left(a_{0}+b_{0}\right)+B^{\prime}
$$

and

$$
\begin{aligned}
A C+B C & =\omega^{\alpha_{0}} a_{0} C+A^{\prime}+\omega^{\beta_{0}} b_{0} C+B^{\prime} \\
& =\omega^{\alpha_{0}}\left(a_{0}+b_{0}\right) C+B^{\prime}=(A+B) C .
\end{aligned}
$$

If $\alpha_{0}>\beta_{0}$, then

$$
A+B=\omega^{\alpha_{0}} a_{0}+A^{\prime}+\omega^{\beta_{0}} b_{0}+B^{\prime},
$$

and

$$
(A+B) C=\omega^{\alpha_{0}} a_{0} c+A^{\prime}+\omega^{\beta_{0}} b_{0}+B^{\prime}=A C+B \leqq A C+B C .
$$

Thus if $0<C<\omega$, then

$$
(A+B) C \leqq A C+B C .
$$

Suppose $C$ is transfinite and limiting, then $\gamma_{l-1}>0$. Let $J=\max$ $(A, B)$; then

$$
(A+B) C \leqq(J+J) C=(J 2) C=J(2 C)=J C \leqq A C+B C .
$$

Suppose $C$ is transfinite and non-limiting, then $\gamma_{l-1}=0$, and if we take

$$
C_{0}=\sum_{i=0}^{l-2} \omega^{\gamma^{i} C_{i},} \quad C_{1}=C_{l-1}
$$

then

$$
C_{0}+C_{1}=C
$$

If $\alpha_{0} \geqq \beta_{0}$, then

$$
\begin{aligned}
(A+B) C & =(A+B)\left(C_{0}+C_{1}\right)=(A+B) C_{0}+(A+B) C_{1} \\
& \leqq A C_{0}+A C_{1}+B C_{1}<A C+B C .
\end{aligned}
$$

If $\alpha_{0}<\beta_{0}$, then

$$
(A+B) C \leqq B C \leqq A C+B C .
$$

Thus if $\gamma_{l-1}=0$, then 


$$
(A+B) C \leqq A C+B C,
$$

and our theorem is proved.

We can also prove the following:

3.2. Corollary. A necessary and sufficient condition that

$$
(A+B) C=A C+B C
$$

for positive $A, B$, and $C$ is that either $C=1$, or $1<C<\omega$ and $\alpha_{0} \leqq \beta_{0}$, or $\omega \leqq C$ and $\alpha_{0}+\gamma_{0}<\beta_{0}+\gamma_{0}$.

This corollary follows quite easily from the reasoning found in the preceding section.

Cornell University

\section{THE DECOMPOSITION THEOREM FOR ABELIAN GROUPS ${ }^{1}$}

\section{JOEL BRENNER}

Let $G$ be an abelian group such that $p^{k} g=0$ for all $g \in G, p$ prime, $k$ fixed. We prove $G$ has a basis, that is, a set of elements such that each $g \in G$ is uniquely expressible as a linear combination of elements of the set. ${ }^{2}$

THEOREM. There exists an ascending chain of sets $B_{i}, 0 \leqq i \leqq k$, of elements of $G$ with the properties:

(i) Every element in $B_{i}$ is of order greater than $p^{k-i}$.

(ii) The elements in $B_{i}$ are completely linearly independent.

(iii) If the order of the element $g$ in $G$ is greater than $p^{k-i}$, then there exists a (unique) linear combination $z$ of elements of $B_{i}$ such that the order of $g-z$ is at most $p^{k-i}$.

Since we may choose as $B_{0}$ the vacuous set, we may assume that the sets $B_{0}, \cdots, B_{s}$ have already been constructed in such a way as to meet the requirements (i) to (iii). In order to construct $B_{s+1}$ we adjoin to $B_{s}$ any greatest subset $C$ of $G$ with the following properties.

(a) All the elements in $C$ are of order $p^{k-s}$.

(b) The join $B_{s+1}$ of the sets $B_{s}$ and $C$ is an independent set.

1 Presented to the Society, April 6, 1940.

${ }^{2}$ Unique in that the number of nonzero terms in an expression for $g$ is unique and only the arrangement but not the respective values of the nonzero terms may differ in two expressions for $g$. 